\title{
AirSTAR: A UAV Platform for Flight Dynamics and Control System Testing
}

\author{
Thomas L. Jordan, ${ }^{*}$ John V. Foster ${ }^{\dagger}$, Roger M. Bailey ${ }^{\ddagger}$ and Christine M. Belcastro ${ }^{\S}$ \\ NASA Langley Research Center, Hampton, VA 23681
}

\begin{abstract}
As part of the NASA Aviation Safety Program at Langley Research Center, a dynamically scaled unmanned aerial vehicle (UAV) and associated ground based control system are being developed to investigate dynamics modeling and control of large transport vehicles in upset conditions. The UAV is a $5.5 \%$ (seven foot wingspan), twin turbine, generic transport aircraft with a sophisticated instrumentation and telemetry package. A ground based, real-time control system is located inside an operations vehicle for the research pilot and associated support personnel. The telemetry system supports over 70 channels of data plus video for the downlink and 30 channels for the control uplink. Data rates are in excess of $200 \mathrm{~Hz}$. Dynamic scaling of the UAV, which includes dimensional, weight, inertial, actuation, and control system scaling, is required so that the sub-scale vehicle will realistically simulate the flight characteristics of the full-scale aircraft. This testbed will be utilized to validate modeling methods, flight dynamics characteristics, and control system designs for large transport aircraft, with the end goal being the development of technologies to reduce the fatal accident rate due to loss-of-control.
\end{abstract}

\section{Nomenclature}

$d_{F S} \quad=$ density of air for full scale vehicle

$d_{M} \quad=$ density of air for model

$K=$ scale factor

$w_{F S} \quad=$ weight of full-scale plane

$w_{M} \quad=$ weight of model

\section{Introduction}

$\mathrm{T}$ he NASA Aviation Safety Program (AvSP) was established to improve the safety of current and future aircraft in the National Airspace System (NAS) by focusing on the design, manufacture, maintenance, and operation of aircraft. Two areas of the AvSP, Integrated Resilient Aircraft Control (IRAC) and Integrated Vehicle Health Management (IVHM), together are working to prevent loss-of-control accidents and to identify and mitigate the contributing factors in these types of accidents. According to the National Transportation Safety Board's accident database, $40 \%$ of all commercial aviation fatalities from 1990 - 1996 were due to loss of control. Technologies being developed include enhanced models of vehicle dynamics to characterize upset conditions, failure detection and identification (FDI) algorithms, and adaptive guidance and control (G\&C) laws. The flight dynamics models for upset conditions have been developed for integration into an enhanced aircraft simulation that is being created for improved upset recovery training, and to support the development and evaluation of the FDI and G\&C algorithms ${ }^{1}$. These algorithms are being developed for use onboard transport aircraft for improved situational awareness and control under adverse and upset conditions related to loss-of-control events. Validation of these technologies is therefore critical. Figure 1 illustrates how an actual loss-of-control accident goes beyond the normal flight envelope into regions where aerodynamic data is not available from conventional sources.

\footnotetext{
* Systems Engineer, Aeronautic Systems Engineering Branch, M/S 238, AIAA member

† Senior Research Engineer, Flight Dynamics Branch, M/S 308, AIAA Associate Fellow.

‡ Senior Research Engineer, Safety Critical Aviation Systems Branch, M/S 130, AIAA member.

${ }^{\S}$ Principle Investigator, Dynamic Systems and Control Branch, M/S 308, AIAA Senior member.
} 


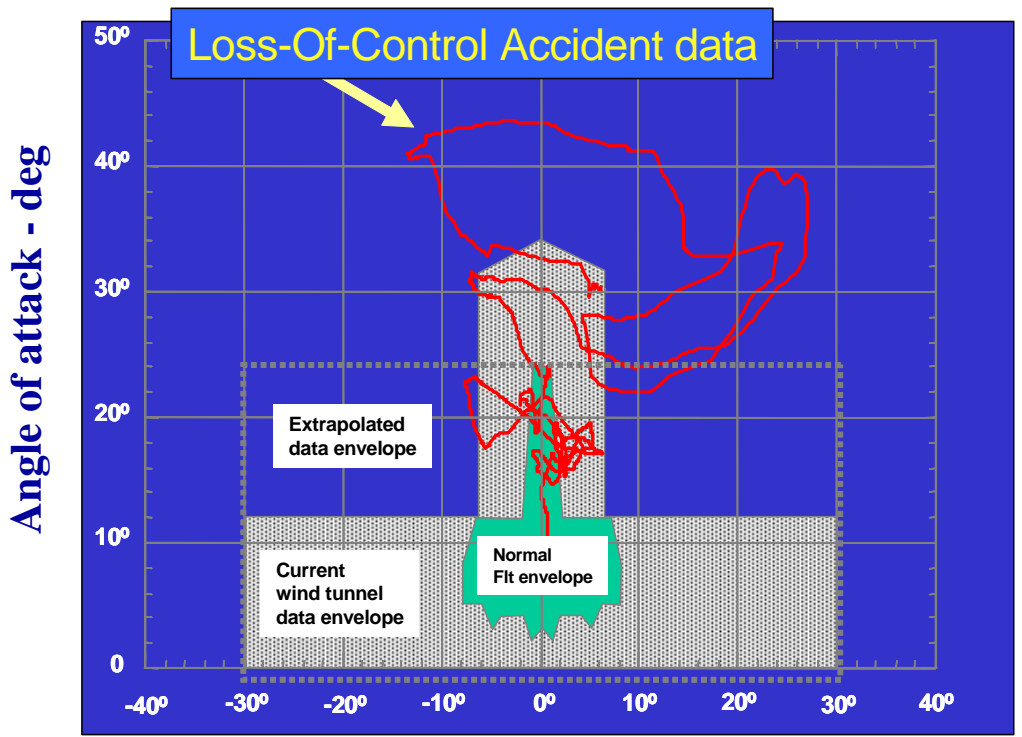

Angle of sideslip - deg

Figure 1. Transport loss-of-control accident relative to angle-ofattack and sideslip.

The AirSTAR testbed has been developed to provide an in-flight capability to validate various flight critical technologies ${ }^{2}$. The testbed is composed of three elements: a 5.5\% dynamically scaled, turbine powered generic transport model $(\mathrm{GTM})^{3}$, a Mobile Operations Station (MOS) and associated ground based facilities $^{4}$, and a test range. This research capability, along with wind tunnel testing, full scale flight testing, and flight simulation, provides the methods and tools to develop and test the technologies demanded by the AvSP.

The expanded flight envelope of the GTM and the requirements to gather large amounts of data (at high rates) presented unique challenges to the development of the AirSTAR testbed. Because the GTM will be operating outside of the normal "benign" flight envelope of full scale transport aircraft and most UAVs, additional measures had to be taken, both on the plane and in the control station, to mitigate the risks associated with this type of flight.

The remainder of this paper is organized as follows: Section II describes the dynamically scaled research model; Section III discusses the Mobile Operation Station and associated ground facilities; Section IV provides insight into the identification of and relationship with the test facility; Section V talks about the research capability of the testbed and Section VI provides some concluding remarks.

\section{Dynamically Scaled Research Model}

\section{A. Model development}

By adhering to the principles of similitude ${ }^{5-6}$, the properties of an object of one size can be mathematically related to the properties of an object of another size. In wind tunnel tests, a subscale airplane can be used to determine aerodynamic characteristics of a full-scale airplane. This same principle can be applied to free flying airplanes with the proper scaling. In wind tunnels, the subscale model must be dimensionally scaled. For free flying models to properly represent full scale flight, they must be dynamically scaled. Dynamic scaling includes not only dimensional scaling, but also scaling for weight, inertia, actuator dynamics, and control system response. For the experiments that the AirSTAR testbed will be used for, such as loss-of-control flight due to high angles-of-attack and sideslip, the flow around the aircraft becomes separated and fluid effects associated with Reynolds number scaling may be minimized ${ }^{1}$. For more benign flight conditions, Reynolds number effects can be significant and the aerodynamics of the model would not be representative of the full scale aircraft for certain maneuvers.

For the GTM, a scale factor, $K$, of 0.055 was chosen based upon the facts that there exists a large data base of wind tunnel data for a 5.5\% model and that a feasibility study had concluded the dynamic scaling requirement could be met (from a structural and payload perspective) at that scale. Based upon this scaling factor, Table 1 shows some characteristic dimensions and attributes of the GTM versus the full scale aircraft. 
Table 1. Comparison of $5.5 \%$ dynamically scaled model and full scale properties

\begin{tabular}{|l|c|c|c|c|c|c|}
\hline & Length & Wingspan & Weight & Roll inertia & Airspeed & Altitude \\
\hline $\begin{array}{l}\text { Full Scale } \\
\text { Transport }\end{array}$ & $145.5 \mathrm{ft}$ & $124 \mathrm{ft}$ & $200,000 \mathrm{lbs}$ & $2.64 \mathrm{e}^{6} \mathrm{sl}_{\mathrm{ft}}{ }^{2}$ & $320 \mathrm{mph}$ & $13000 \mathrm{ft}$ \\
\hline $5.5 \%$ Model & $96 \mathrm{in}$ & $82 \mathrm{in}$ & $49.6 \mathrm{lbs}$ & $1.33 \mathrm{sl}^{-\mathrm{ft}^{2}}$ & $75 \mathrm{mph}$ & $1000 \mathrm{ft}$ \\
\hline
\end{tabular}

A correction is made to the weight of the model since the altitude (and therefore density of the air) at which the model will operate is not the same as the operating altitude of the full-scale plane. This corrected model weight can be expressed as:

$$
w_{M}^{\prime}=K^{3} w_{F S} \frac{\left(d_{M}\right)}{\left(d_{F S}\right)}
$$

Once the model had been sized and the weights and inertias determined using the above scaling parameters, then the functional requirements of the model could be determined. These include parameters such as flight time, battery and fuel capacity, payload, data system requirements, structural factors of safety, rigidity, symmetry, and control surface deflection. The airframe was designed using Pro-Engineer Solid Model software allowing the designers to generate a solid model representation of the desired 5.5\% geometry with associated mass properties for structure and sub-systems. This started with the creation of solid model parts and sub-assemblies in an attempt to create all components, whether they be structural or commercial-off-the-shelf (COTS), needed for a complete design. All of the vehicles components were modeled as accurately as possible with regard to geometry, center of gravity, and weight. Sub-assemblies were then generated from these individual components to represent the wings, the fuselage, the landing gear units, the pneumatic system, etc. A final assembly was generated by combining all of the major components, the minor components, and the sub-system sub-assemblies. As the individual parts were fabricated and assembled, the ProEngineer Model was updated for current weights and sizes. This level of detail includes all required fasteners, adhesives, primer, and paint to accurately estimate the vehicle weight and inertias. After assembly of the vehicle, the weight and inertias were measured in the laboratory and found to be within $0.1 \%$ for the weight and within $3 \%$ for the inertias of the estimated values. By having this level of fidelity in the solid model, the designers were able to quickly determine the effect of adding components or moving them around in the airframe with respect to the weight and inertia targets. Figure 2 shows the solid model of the T2 research airframe.

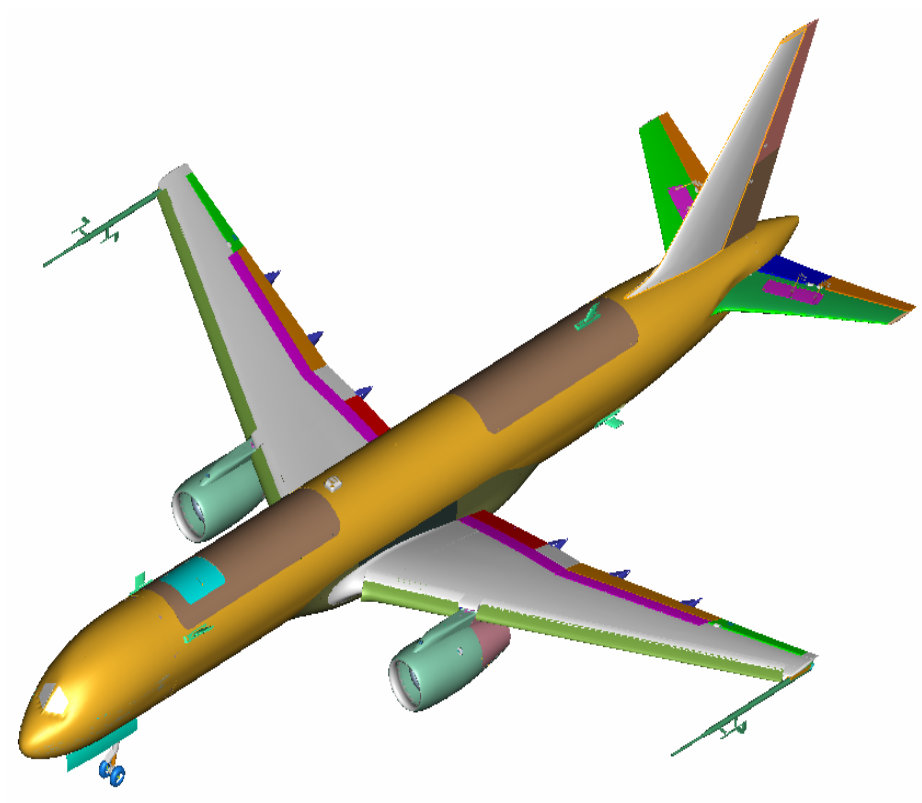

Figure 2. The 5.5\% GTM-T2 solid model 


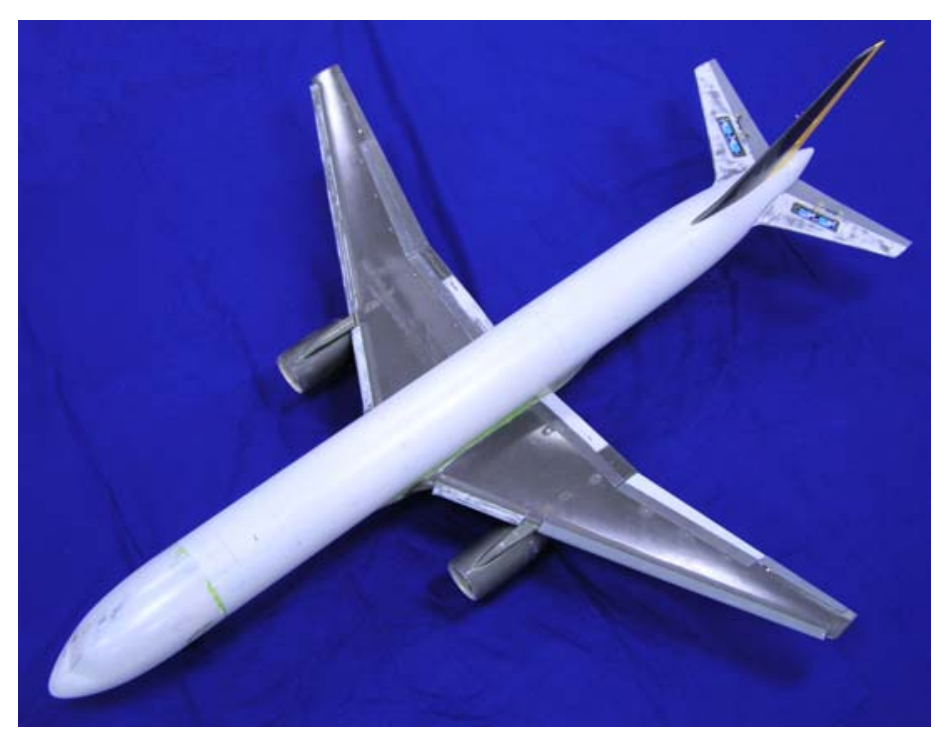

Figure 3. The completed T2 airframe
The research model, shown in Fig. 3, was fabricated in the NASA Langley Composite Models Development Shop. The construction of the model consisted of a fiberglass and honeycomb sandwich composite for the fuselage with carbon used on the wings and empennage. Carbon was used to reduce weight and increase stiffness and strength. Carbon was also used on the main wing spar and some substructure internal to the fuselage. This weight savings, due to the incorporation of carbon into the structure, resulted in a lighter airframe and increased payload capacity for research equipment. A similar training model (T1) was made using fiberglass instead of the carbon. The resulting airframe was 30\% heavier and therefore did not have the payload capacity for the research data and control system. The T1 aircraft has been used extensively for pilot training exercises.

\section{B. Onboard data and control system}

To accomplish the research objectives ${ }^{7}$, a suite of sensors, actuators, instrumentation, and controls were identified. The $5.5 \%$ scaling factor afforded the design team the ability to utilize many commercial-off-the-shelf (COTS) components in the airframe, including actuators, turbine engines, landing gear, batteries, and electronics. From scaling and similitude requirements, the resulting subscale model response is faster than a full scale model by a factor of $\sqrt{K}$. For a 5.5\% model, this corresponds to an increase in response and control of 4.25 times that of the full scale aircraft, and just over $200 \mathrm{~Hz}$ for the fastest data rates. Because of the ambitious real-time computing requirements, the limited space and weight budget of the model, and the inherent risk in flight beyond the normal envelope, it was determined that the plane would utilize a ground based flight control system for research maneuvers. This decision to remotely control the airplane had both benefits and drawbacks. The benefits included removing the flight control system from the cramped and environmentally harsh environment inside the model which also eliminated the risk of losing the system in the event of a crash. However, this placed additional requirements on the telemetry system to deliver data to the ground and send control signals back to the plane with minimal interruptions. This air and ground based control and data system is discussed in more detail in Section III.

Downlink parameters required for research include control surface position (measured by potentiometers on all control surfaces), differential GPS position and velocities, pressure derived airspeed and altitude, magnetic heading, attitude, rates, nose camera video, turbine throttle and RPM, and various system health monitoring signals such as battery voltage. Uplink parameters include control surface deflection and throttle position commands, landing gear extend/retract commands, safety or research pilot control, and DGPS corrections from the ground station.

\section{Operation}

In an effort to reduce the risk that comes with operating a UAV, and more specifically one that is flying at unusual attitudes, the project instituted a risk mitigation program to train safety pilots and to migrate flight hardware from low risk platforms to the research aircraft. In the nominal operational concept of the AirSTAR testbed, a safety pilot with conventional RC hardware will take off the GTM, switch control to a research pilot based in the MOS who will conduct the research maneuvers, and then take back control of the plane for landing. This safety pilot will be located outside of the MOS and shall maintain visual contact with the plane. The safety pilot will have the ability to take control (by means of a command sent to the onboard safety switch) of the aircraft at any time during the flight should the research pilot have difficulty controlling the plane. This safety switch is the device that passes control between the safety and research pilots. In the event of a loss of signal, the safety switch can also direct control of the airplane to an onboard autopilot or default to a preprogrammed failsafe condition. 
The basis of the project's risk reduction plan, shown in Fig. 4, is the development of a stable of remotely piloted aircraft that start with commercial off-the-shelf (COTS) propeller and ducted fan powered platforms (Phase 1), then advance progressively through single turbine COTS trainers (Phase 2), and finally to custom, dual turbine, swept wing platforms including dynamically scaled transports (Phase 3). The project designed and built two dynamically scaled aircraft. The first, designated T1, is used strictly for pilot training and carries the minimum amount of telemetry and control electronics. The second, designated $\mathrm{T} 2$, is the research aircraft. It has a lighter airframe which makes a heavier payload possible for the data gathering, telemetry, and control electronics required for the research experiments. In addition to mitigating risk by utilizing different types of aircraft, the plan also addressed wing loading as a factor in training. The $\mathrm{T} 1$ and $\mathrm{T} 2$ aircrafts have a wing loading of approximately 142 $\mathrm{oz} / \mathrm{ft}^{2}$. Trainer aircraft were loaded with weight to progress to this value. This strategy of increasingly complex,

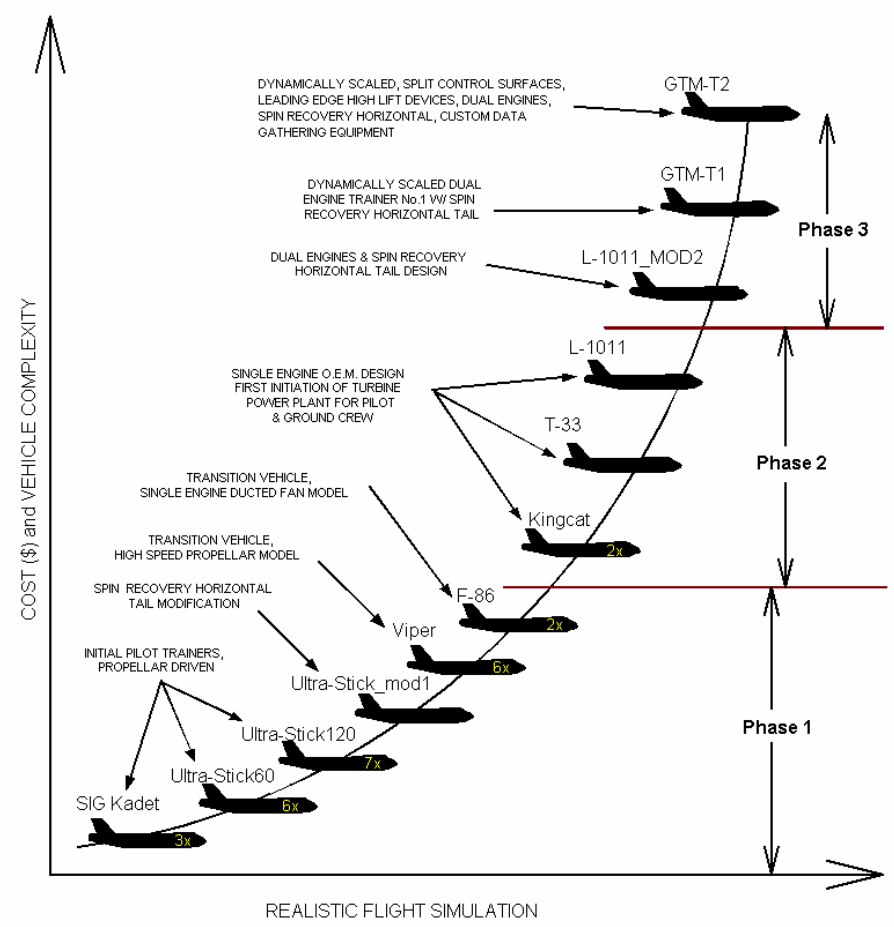

Figure 4. Airplanes used in pilot training program sophisticated, and expensive aircraft provided a path for the GTM team to gradually but steadily enhance its proficiency to fly and operate subscale turbine aircraft.

Using data from previous wind tunnel tests along with as-built mass properties data from the fabrication team, a non-linear six degree-of-freedom flight dynamics model of the GTM was developed and incorporated into a realtime pilot simulation tool. This PC-based simulator was used by the pilots to evaluate the flight handling characteristics of the dynamically scaled aircraft. It also allowed the pilots to practice flight procedures during degraded performance conditions.

\section{Ground Facilities and Mobile Operations Station}

Use of a Remotely Piloted Vehicle (RPV) for conducting flight tests to validate control upset prevention and recovery technologies (including modeling and control methods for characterizing and recovering from upset conditions) requires a robust and reliable ground support system for pilots and researchers. This section describes the ground facilities being developed to support the AirSTAR testbed. Figure 5 shows a functional diagram of the basic GTM-Ground Facilities (GTM-GF) architecture. 


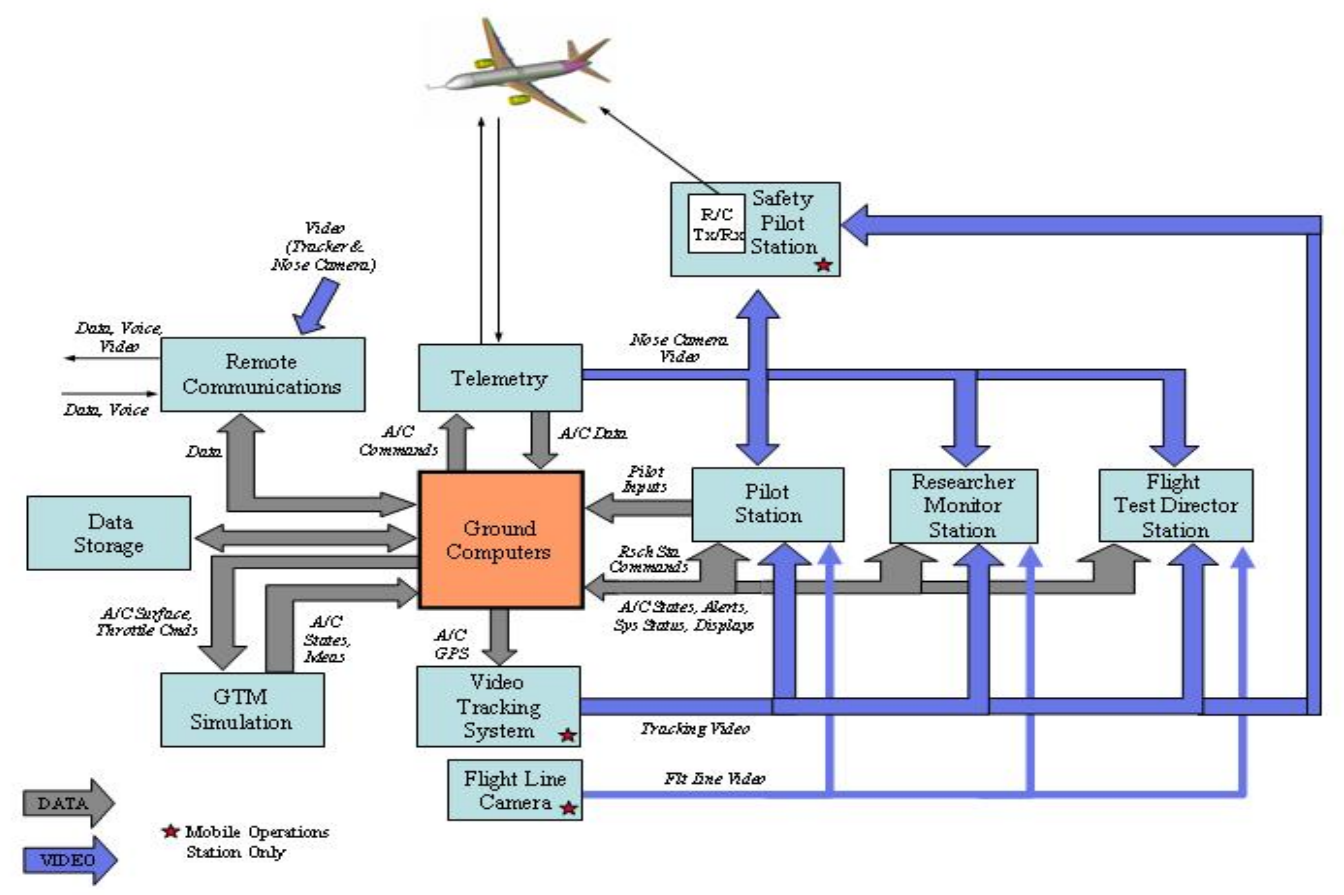

Figure 5. Block Diagram of Ground Facilities Functionality

The fundamental purpose of the GTM-GF is to provide the tools to accomplish the following:

- $\quad$ Permit research pilots to control the GTM;

- Receive, process, record and transmit GTM telemetry data;

- Display information to pilots, researchers, systems engineers and observers;

- Provide voice communications for experiment participants and observers;

- Provide data processing systems capable of computing control parameters on the ground to augment pilot control and/or remotely drive aircraft control surfaces;

- $\quad$ Provide real-time GTM simulation for training, testing and experiment reproduction.

The GTM Ground Facilities are designed to align with the overall development of the Systems and Airframe Failure Emulation, Testing and Integration (SAFETI) Research Laboratory. The SAFETI Lab is being developed for validation testing of AvSP technologies and to support long-term experimental research goals and objectives.

The GTM-GF will ultimately be the conjugation of two separate resources: (1) the Mobile Operations Station (MOS) - a self-contained, motorized vehicle serving as a mobile research command/operations center, capable of deployment to remote sites when conducting GTM flight experiments; and (2) the Base Research Station (BRS) - a laboratory based at the NASA Langley Research Center (LaRC) providing near-identical capabilities as the MOS. Figure 6 below provides an overview of the GTM-GF Hardware Systems including the BRS/MOS interaction. 


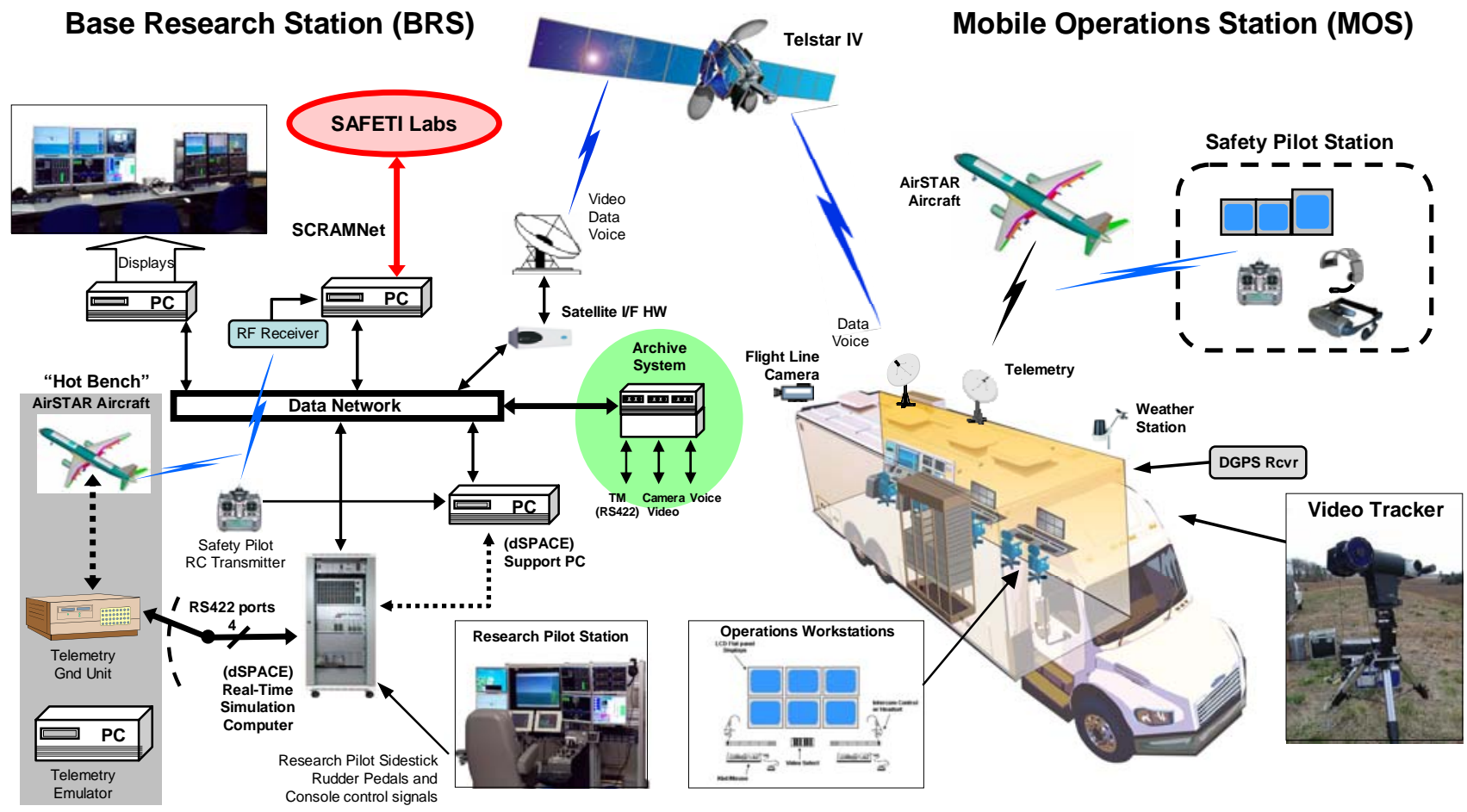

Figure 6. GTM-GF Hardware Systems Overview

The overall GTM-GF architecture will also include the capability to link the BRS and MOS (data, video and voice) during GTM flight experiments. The BRS will be used for experimental software development and testing and includes a "hot bench" work area which will accommodate hardware-in-the-loop test and evaluation with the GTM. The GTM-GF is comprised of three major hardware subsystems: the air-ground data link, the computational resources, and the user interfaces, all described below.

\section{A. Air-Ground Data Link}

Communications between the AirSTAR aircraft and the GTM-GF are via a duplex serial data link using microwave RF transceivers, with a separate safety-pilot backup command RF link. To maintain effective telemetry communications coverage, the GTM-GF will employ a directionally-tracked, circularly-polarized antenna system. Analysis of the anticipated RF budget (involving transmitter powers, receiver noise floors, and antenna gains) supports the desired operation of the microwave links to slant ranges up to 2 statue miles with power margins in excess of 15 decibels. The AirSTAR telemetry system evolved from an air-to-ground data link system and utilizes a proprietary encoding scheme based on the IRIG-106 standard. The telemetry system is comprised of a custom built Telemetry Airborne Unit (TAU) installed in the GTM and a custom built Telemetry Ground Unit (TGU) interfaced to the GTM-GF. The on-board TAU is microprocessor-based and incorporates hardware to assist in aircraft operations and instrumentation, including signal interface boards, safety switch, and power supplies. The TAU acquires data from various sensors aboard the GTM, including potentiometers indicating control surface position, angle-of-attack probes, engine control units (ECU), an inertial navigation system, battery status, and employs a 'Flight Controller' module to multiplex this data for the air-to-ground telemetry stream. Conversely, the 'Flight Controller' module is also used to de-multiplex the ground-to-air telemetry stream, distributing commands to GTM subsystems, including the flight surface servos. Additionally, video from the GTM's nose camera will be transmitted via the RF link to the ground.

The TGU's primary function is to perform the multiplexing and de-multiplexing of the telemetry data for GTMGF client computers, and is housed in a standard 19” rack-mountable enclosure. The TGU interfaces to the GTMGF computing system via four asynchronous serial (RS-422) ports, each running at 115,200 baud, that are used to receive flight data from the GTM and transmit servo and throttle commands to the GTM. 


\section{B. Computational Resources}

Scaled flight research has demanding data system frame timing deadlines that require the deterministic program execution rate provided by a real-time operating system (RTOS). The GTM-GF computing system is capable of accomplishing three primary tasks:

1. Receiving telemetered GTM state data (e.g. sensor outputs, discretes, etc.), converting these data to calibrated engineering units in real-time, and transmitting control-surface and engine commands and other required state data to the GTM via the telemetry system;

2. Processing, in real-time, researcher-provided algorithms, including

a. flight control algorithms to compute control-surface and engine commands in response to pilot inputs and/or aircraft state data;

b. failure detection and isolation (FDI) algorithms, upset recovery algorithms, and guidance algorithms;

3. Providing real-time, hardware-in-the-loop (HIL) simulation of the GTM, including the capacity for integrating synthetic vision display components and terrain databases for flight control by the research pilot.

Non-time-critical computing tasks such as generation of user displays are managed by Intel ${ }^{\circledR} /$ Windows ${ }^{\circledR}$-based PCs. Time-critical computing tasks will be managed by the dSPACE ${ }^{\circledR}$ computing platform. dSPACE, which integrates target processor hardware, $\mathrm{C}$ code, analog and digital input/output, and control law block diagrams, was chosen because it was based on Matlab ${ }^{\circledR}$ and Simulink ${ }^{\circledR}$ products developed by The Mathworks, Inc. The dSPACE platform is a good fit with existing commercial aircraft simulations based on tools developed by The Mathworks, Inc. Matlab's plotting and scripting features facilitate manipulation of wind tunnel data sets to obtain aerodynamic data parameters and verify control algorithms against check data. The graphical data flow characteristics of Simulink are a close fit to the characteristics of control law diagrams in aerospace documentation. The use of dSPACE reduces the amount of custom programming necessary to integrate the aircraft telemetry subsystems. The commonality between dSPACE and the simulation tools facilitates exercising the software at each stage of development with a realistic execution environment, and rapidly testing various HIL configurations.

\section{User Interfaces}

GTM-GF operations will be conducted by personnel located at three primary work areas and one external work area: the Flight Research Station, the Operations Command Station, Operations Engineering Station, and the Safety Pilot Station. Additional user subsystems include data archive, audio/video, satellite data link, and a video tracker. Each of these work areas and subsystems are described below.

\section{Flight Research Station (FRS)}

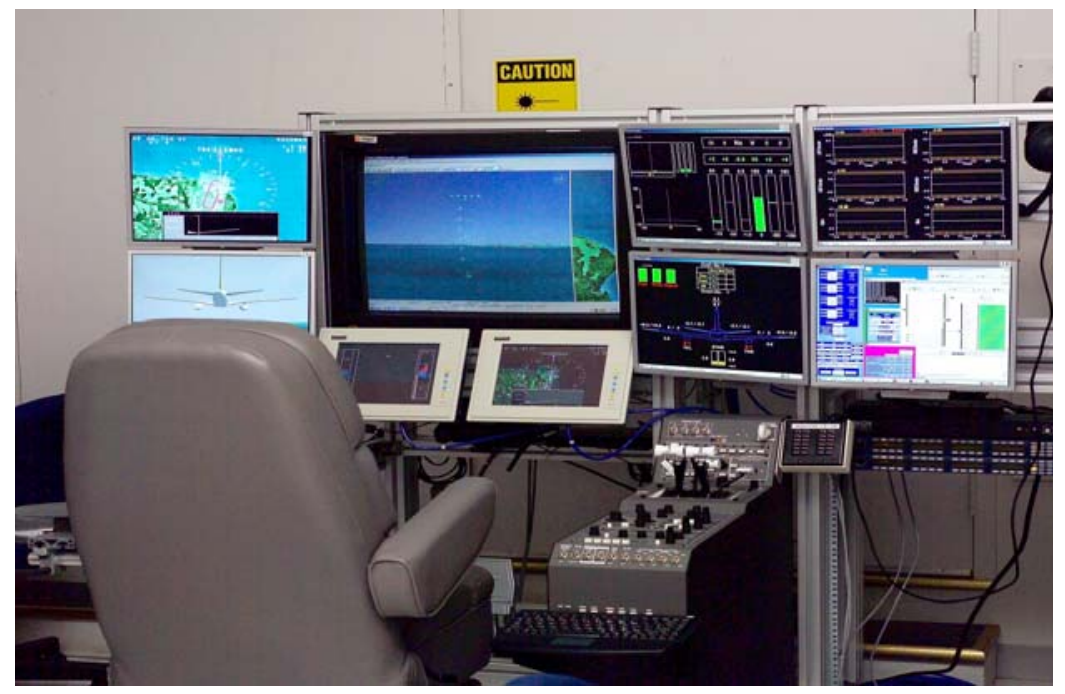

Figure 7. Flight Research Station in BRS
The GTM pilot and research project's principal investigator (PI) will be located in the FRS. This station provides the means to fly the GTM manually or automatically by engagement of a researcher-provided autopilot flight control system implemented in the dSPACE computer. The FRS will provide all necessary pilot controls and a variety of displays for the pilot and PI. Figure 7 shows a photograph of the FRS. 


\section{Operations Command Station (OCS)}

The Flight Test Director, who has the responsibility of coordinating all flight test related activities, and another researcher will be located in this area. The OCS will be provided with six display units, and the capability to configure and select among all available video sources as desired. The OCS will provide for monitoring and control of all audio communications between flight test participants. The OCS will also provide the capability to conduct experiment-specific activities such as:

- $\quad$ Selecting test parameters for display;

- Setting test parameters that control test conditions;

- Setting simulated fault conditions;

- Performing pre/post-flight analysis of recorded flight data, including running the GTM simulation.

\section{Operations Engineering Station (OES)}

Hardware and software support engineers will be located in this area. These support engineers will be responsible for monitoring and maintaining GTM-GF hardware and software subsystems during flight test operations. The OES will be similar in appearance to the OCS, and will have keyboard and mouse access to all GTM-GF computers, as well as the satellite terminal and data network. Figure 8 shows the general layout for the OES and OCS.

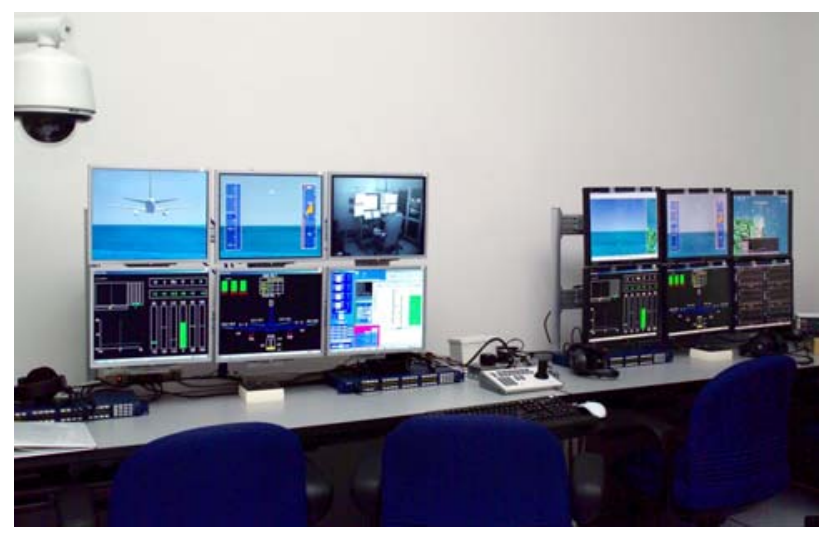

Figure 8. Operations Engineering Station (left) and Operations Command Station in BRS

\section{Safety Pilot Station}

The safety pilot is located externally to the MOS vehicle to provide a line-of-sight view of the GTM so as to afford direct observation of aircraft attitude when possible. If safety pilot control is required when the GTM is beyond the line-of-sight, the safety pilot will use displays of the nose camera video, tracker camera video, or a combination of the videos to fly the GTM back into line-of-sight and safely land the airplane. The safety pilot also has a dedicated two-way audio communication channel with the Flight Test Director Station and the Research Pilot Station.

\section{Other Subsystems}

In addition to the primary work areas and Safety Pilot Station, GTM-GF users will interact with the four main subsystems described below.

DATA ARCHIVE SYSTEM - Integral to the mission of AirSTAR scientific research is data collection and storage. The success of post-flight analysis hinges on the accurate and properly registered capture of GTM state parameters and GTM-GF process elements. With valid and complete data, the stated goals of investigating new aerodynamic technologies can be more fully realized. The GTM-GF is equipped with an Asynchronous Real-Time Multiplexer and Output Reconstructor (ARMOR) subsystem. The multiplexing system merges multiple data sources, including GTM PCM telemetry data, $10 \mathrm{Mb} / \mathrm{s}$ Ethernet, voice, time code signals and video into a composite stream for digital recording. A plug-in Recorder Interface Module (RIM) provides a link to the digital recorder unit, which provides a recording interface to RAID 1 hard drives, with $90 \mathrm{Mb} / \mathrm{s}$ data transfer rate. This ARMOR system is configured to provide playback of all recorded GTM flight data parameters, physical camera video outputs, synthetic display video 
outputs, audio loops, and UTC time tag information, with partial or complete data reconstruction, including downlink/uplink telemetry to the GTM, camera video, audio loops, and master UTC information, allowing new realtime simulations to execute using recorded state data. Partial reconstruction provides the capability to perform engineering tests of the air-ground system, and debugging of anomalous behavior.

AUDIO/VIDEO SYSTEM - The GTM-GF provides the communications infrastructure to allow all key participants in GTM flight test activities to maintain voice contact. The heart of the intercom system is the ClearCom Inc. MicroMatrix ${ }^{\circledR}$ 24-channel digital intercom system. Onboard slots for interface cards permit seamless interfacing with telephones, two-way radios, party-line intercoms, and wireless belt packs. Set up of point-to-point communications, groups, monitoring lines, and "virtual" party lines for all flight test participants is accomplished using interactive and menu-driven Windows-based software. The intercom master control system is operated by the flight test director during GTM flight test operations.

The GTM-GF provides the video hardware infrastructure to allow all video camera output signals and all computer generated displays to be amplified and split as necessary for distribution to key participants in GTM flight test activities. The heart of the GTM-GF video distribution system is Extron Electronics' CrossPoint Plus 24x24 matrix video switch, an ultra-wideband analog RGBHV matrix switcher, capable of distributing any of 24 video inputs to any combination of 24 video outputs simultaneously. Additionally, a controller is interfaced to the video matrix switch, permitting users to operate the matrix switch from the Flight Research Station, Operations Command Station or the Operations Engineering Station.

SATELLITE DATA LINK SYSTEM - In order to provide the capability to monitor MOS GTM flight test activities at the Base Research Station located at Langley Research Center, a satellite backbone transmission system will be utilized to (1) transmit GTM telemetry state data, optical video and voice communication from the MOS to the BRS, and (2) transmit desired support data and voice communications from the BRS to the MOS. The major communications system elements comprising the satellite system consist of a fixed base station satellite dish, a deployable remote station satellite dish, and the necessary interface equipment. The base and remote stations employ a satellite backbone transmission system augmented by data, video and voice communication facilities, and an Internet Protocol (IP) routing system. The IP routing system provides for multiple communication transmission types and automatic routing path requirements. The base station consists of a 2.4 meter dish antenna and rack mounted satellite, video, audio, and routing equipment. The remote station consists of a 1.2 meter dish antenna contained in two field carrying cases, a field case for the antenna RF equipment, and satellite, video, audio, and routing equipment mounted in a separate field carrying case. The remote station is further augmented by a wireless video and audio transmission system, which provides for line of sight transmission of tracking video and voice communications from the video tracking station.

VIDEO TRACKING SYSTEM - In order to provide the capability to monitor the GTM in flight, a ground-based video tracking system will be deployed with the MOS and will track the airborne vehicle using cameras and electronic equipment. The tracking video could be used by safety pilots to help fly the GTM back to the MOS if a takeover is required when the GTM is beyond the line-of-sight. This video tracking system will be augmented by a wireless video and audio transmission system to provide for transmission of tracking video and voice communications from the video tracking system to the MOS. Tracker slew rate limitations dictate that setup will be at a location one to two miles from the MOS. The setup also requires an operator to be present. The tracking system is capable of utilizing GTM GPS/DGPS position data from the MOS via a wireless modem in order to augment the system's ability to maintain GTM tracking lock.

\section{Mobile Operations Station (MOS) and Simulation}

In addition to the hardware subsystems, work areas, and user subsystems described in the preceding sections, two aspects of the GTM-GF will be described: the Mobile Operations Station and the GTM Simulator.

MOBILE OPERATIONS STATION - The Mobile Operation Station, shown in Fig 9, is a deployable remote laboratory that will be employed as the central research and command center for GTM research flight operations. In addition to all of the hardware and software components utilized in the BRS, the MOS contains the ancillary support subsystems required for operation in the field, including a $60 \mathrm{~kW}$ portable generator, an uninterruptible power supply (UPS) back-up subsystem, four full and two half size equipment racks, restroom facility, kitchenette, small meeting/work area, walk-on roof with safety rails, and leveling jacks. In addition to all the interfaces available in the BRS, the MOS provides external power and data interfaces for additional subsystems, including the safety pilot station, video modem and flight line video camera, wireless transmitter for GPS data, VHF and cellular communications devices, LAN/internet, 1.2 meter portable satellite dish, and 120VAC power outlets. The unit can either be powered by the portable generator or from shore power if available. 


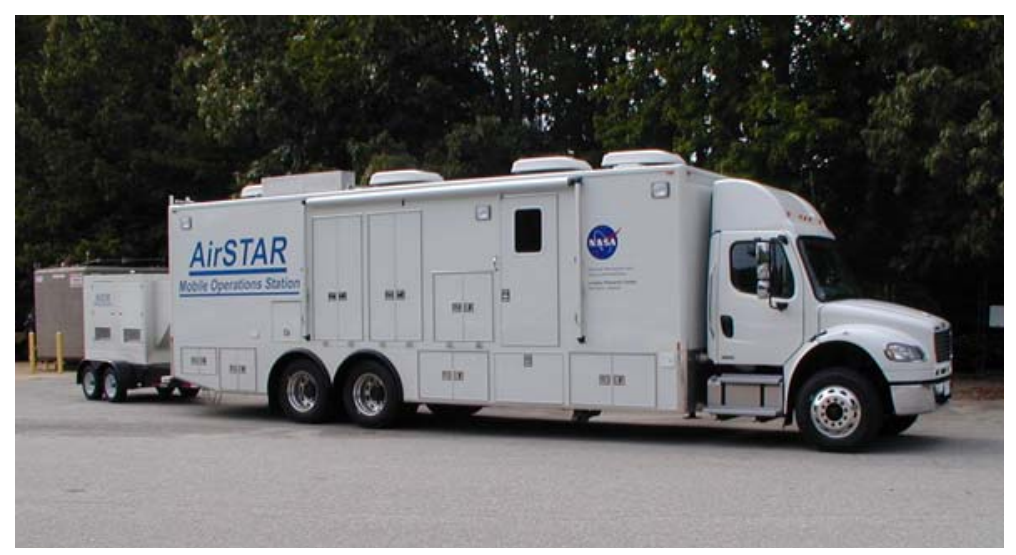

Figure 9. Mobile Operations Station with portable generator

GTM SIMULATION - The GTM flight simulation is capable of being executed in both the BRS and the MOS, and will acquire pilot and researcher input signals and generate six degree-of-freedom (6DOF) flight dynamics and sensor data and emulate the GTM down-link telemetry stream. The simulation will run on a Personal Computer (PC) platform with the Windows operating system, using the Matlab/Simulink programming tools. The GTM simulation is referred to as the Flight Dynamics Model (FDM), and will operate in three distinct configurations:

- Personal Computer (FDM-PC), standalone, near real-time operation, on one or more PCs

- Piloted Simulation (FDM-PSIM), real-time simulated operation on the dSPACE platform

- Hardware In the Loop (FDM-HIL) real-time operation simulation on the dSPACE platform

\section{Test Range}

\section{A. Requirements}

The third component of this testbed is the facility at which the research experiments will be carried out. The identification of this facility and the development of working relationships with the personnel at the test site early in the project are critical to the timely commencement of a research program. Once the research requirements have been finalized and a test vehicle and supporting equipment have been identified, then the requirements for a test facility can be developed. Some of these requirements will be based on the testbed and the specific research plan, such as runway length and width, range size, available infrastructure of the facility, remoteness, airspace type, etc. Other factors may be programmatic and include factors like facility cost, travel and lodging costs, and demand for the range from other users. For the AirSTAR testbed, the project identified the UAV Runway on Wallops Island at NASA Wallops Flight Facility to conduct its research experiments. This facility provides controlled access, a sufficient runway and range to fly the research experiments, restricted airspace, adequate infrastructure, a cooperative and helpful staff, and fits within the project budget. Some of the services provided by the facility include interfacing to the Federal Aviation Administration (FAA) and local fire station, weather monitoring and forecasting, RF signal monitoring, and access control. Figure 10 is an aerial photo of the existing $750 \mathrm{ft}$ runway which is currently being extended to $1500 \mathrm{ft}$. 


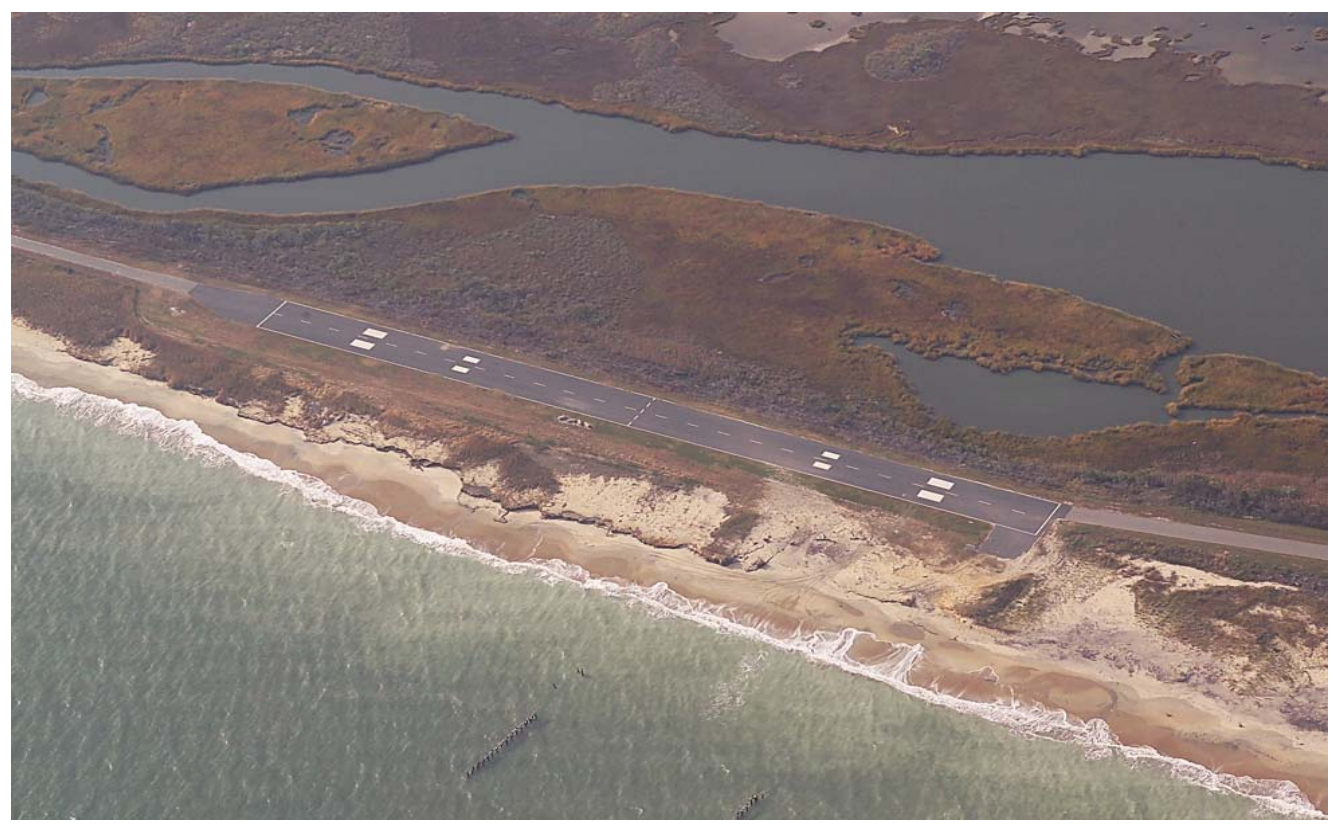

Figure 10. UAV runway at Wallops Island

\section{B. Additional Test Sites}

In addition to the UAV runway at Wallops Island, the project was able to acquire the use of a private runway and airfield within a short distance of LaRC at which to conduct pilot training exercises and checkout flights of various hardware and software packages. The availability of this field and its proximity to LaRC enables the project to operate there several times per week and continue to make progress in a timely fashion towards the goal of conducting the research experiments. Since this facility provides minimal infrastructure and staff, and operations there are in the public airspace, it is not be suitable for conducting the research experiments.

\section{FAA coordination}

Because of the explosive growth of the UAV market worldwide, regulatory agencies and individual test facilities have had to quickly develop standards and regulations to oversee the safe operation of UAVs. This has resulted in ambiguous and sometimes conflicting guidance to the UAV community. The FAA makes a distinction between model airplanes and UAVs and sets forth certain safety standards ${ }^{9}$ for the operation of model aircraft; however most of the oversight for model aircraft is left to the Academy of Model Aeronautics (AMA). For UAVs operating in the NAS, the operator must follow FAA guidance ${ }^{10}$ and apply for a Certificate of Authorization (COA). For the AirSTAR project, this entailed filing the COA application, waiting for approval, and abiding by the restrictions set forth in the COA. Currently the COA is good for one year and is specific to type of aircraft and location. Renewals should be applied for six months in advance of the expiration. If operating in restricted airspace, the above guidelines do not apply.

\section{Experimental Capability}

\section{A. Description of Experimental Capabilities}

The AirSTAR system is designed to conduct aerodynamic and control system experiments relevant to large transport category airplanes. The flight vehicle is unique in that the airframe is based on a modern commercial airplane and it is designed to replicate important flight characteristics of the full-scale vehicle. An important advantage of this test vehicle is that it allows flight investigations in regions of the flight envelope that are not safe to test on the full scale manned vehicle. An additional advantage is the significantly reduced cost compared to flying the full scale vehicle. 
The AirSTAR vehicle is considered part of a suite of tools for conducting control upset prevention research. These tools include predictive methods, such as wind tunnels and simulations, as well as control system analysis and design methods. Typically, simulations are based on wind-tunnel-derived aerodynamic data that are mathematically modeled for use in the equations of motion. However, accurate modeling in regions of the flight envelope characterized by high wind angles, high angular rates, and separated flow, is a formidable challenge and the literature on modeling large transport airplanes in upset conditions is very limited. Because wind-tunnel-based data may not completely emulate the full-scale flight characteristics, the AirSTAR vehicle allows evaluation and validation of the simulations that are critical for accurate control system development and technology demonstrations. For example, certain wind tunnel test methods involve measuring the effects of vehicle motion (e.g. body-axis rates) on aerodynamic forces and moments. These methods include the use of oscillatory and steady motion test rigs but they do not fully account for all flight motions, such as multi-axis or variable frequency maneuvers, that can occur in out-of-control flight. Therefore, flight vehicles, such as AirSTAR, fill a critical need to study highly dynamic flight motions in loss-of-control flight regimes.

The experiments intended for the AirSTAR vehicle include measurements of aerodynamic forces and moments in off-nominal and loss-of-control flight regimes, automatic recovery and guidance control system demonstrations, and validation of advanced control system algorithms for flight control failures. Future plans include airframe modifications for the purpose of structural and aeroelastic testing in off-nominal and damage scenarios.

\section{B. Initial Experiment}

The Dynamics Modeling Experiment (DMEx) is the first flight test program planned for the vehicle and this experiment is intended to validate and update aerodynamic models developed from wind tunnel testing conducted under the NASA AvSP ${ }^{7,11-14}$. Because the flight vehicle is the identical scale as the wind tunnel model, this experiment will focus on the mathematical representation of aerodynamic characteristics in upset conditions without the need to consider scale effects. However, the ultimate goal of this research is to develop methods that predict full-scale dynamic responses using the subscale based measurements.

The experimental approach is based on the previously discussed principles of similitude, often referred to as "dynamic scaling", whereby scaling laws are used to allow prediction of full scale dynamic behavior from sub-scale vehicle testing. The DMEx experiment relies on measurement of rigid-body dynamics using aerodynamic and mass scaling. Full-scale aerodynamic measurements are predicted when the subscale vehicle is operating at identical wind angles (angle of attack and sideslip), control deflections, Reynolds number and Mach number. However, it is well understood that simultaneously matching Reynolds number and Mach number is difficult and must be taken into consideration when designing flight experiments and analyzing flight data. The scaled dynamic responses are predicted by when the aerodynamic scaling is satisfied and the mass properties are scaled via the relative density ratio and Froude number similitude. The mass scaling is achieved by building the flight vehicle to specific angular inertias and weight for a desired test altitude and Froude scaling allows time history prediction from the flight data. As a result, the $5.5 \%$ scale vehicle angular rate response is 4.25 times faster than the full scale vehicle but the airspeed is a factor of 4.25 times slower.

The DMEx test approach will involve measurements of the flight vehicle's linear and angular accelerations for a variety of maneuvers and flight conditions. Properly designed test maneuvers will allow measurements of aerodynamic forces and moments as a function of wind angles, angular rates, and control positions. State-of-the-art system identification methods ${ }^{15}$ will be used to quantify and model various aerodynamic properties and used to perform model updates as the flight envelope is expanded. These data will be compared to existing aerodynamic models developed from wind tunnel data to assess and update the mathematical model structure and identify additional data dependencies or model requirements. Table 2 shows the types of flight test maneuvers that will be conducted in the DMEx flight testing.

Table 2. Experiment maneuvers

\begin{tabular}{|l|l|}
\hline \multicolumn{1}{|c|}{ MANEUVER } & \multicolumn{1}{c|}{ DATA } \\
\hline $\begin{array}{l}\text { Control surface inputs (steps, } \\
\text { doublets, frequency sweep) }\end{array}$ & Control effectiveness, static and dynamic stability \\
\hline Steady 1g trim & Pitch axis static stability and control \\
\hline Steady sideslip & Directional axis static stability and control \\
\hline Approach to stall & Lateral-directional stability, aerodynamic asymmetries \\
\hline Stall and departure & Departure susceptibility, unsteady flow characteristics \\
\hline Post-stall gyrations/spins & Multi-axis dynamics, separated flow characteristics \\
\hline
\end{tabular}


The overall test approach will be a phased buildup in risk considering the potential for entering loss of control flight. Figure 11 illustrates the buildup approach with increasing wind angles and risk for loss-of-control.

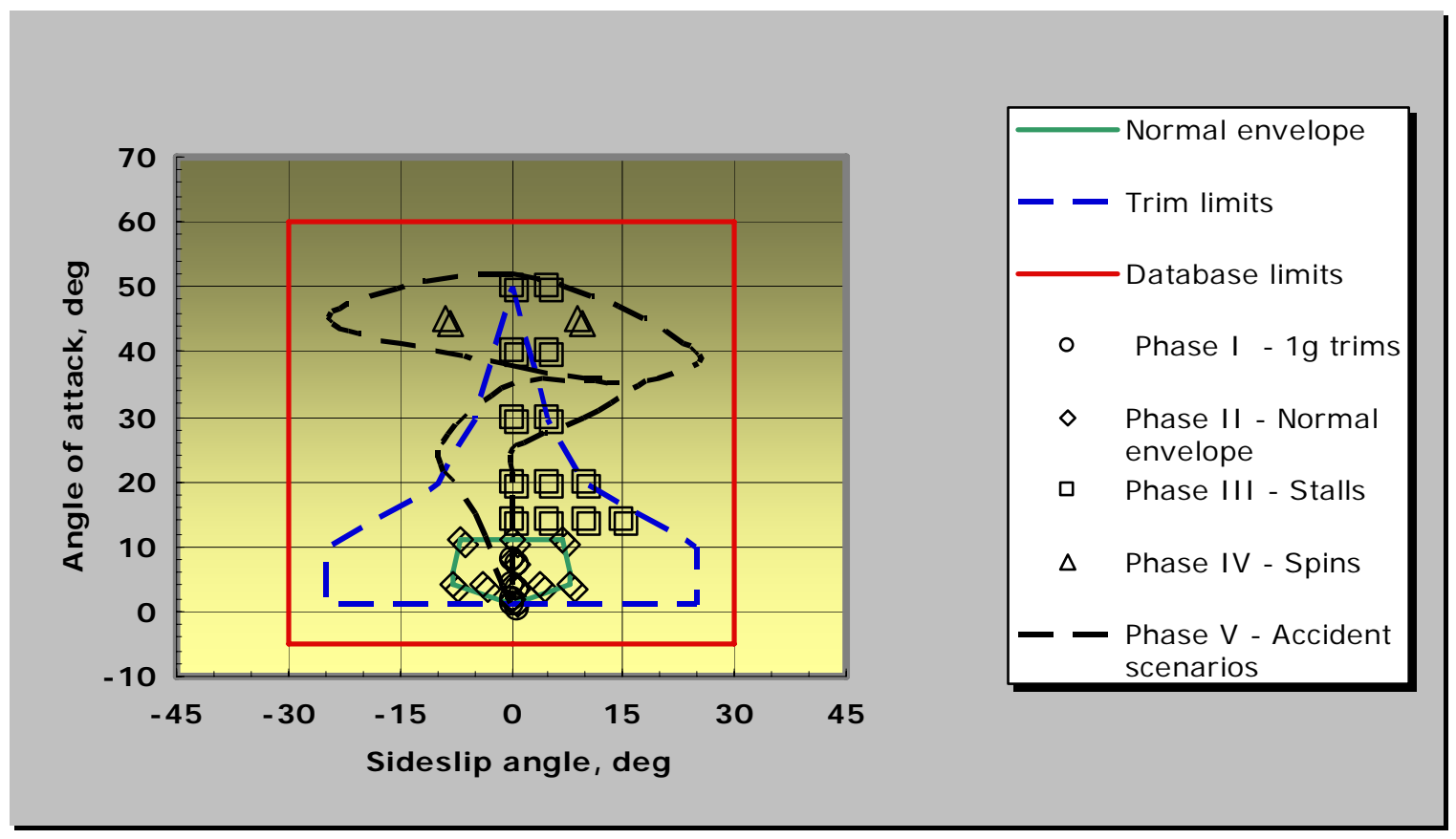

Figure 11. Illustration of phased buildup approach

A critical requirement of the AirSTAR system is the accurate measurement of flight conditions and vehicle response for all maneuvers. Air data include measurements of angle of attack and sideslip, static and total pressures for barometric altitude and calibrated airspeed, linear and angular body-axis accelerations, and all control positions. For example, goals for measurement accuracy of angular rates are $0.05 \mathrm{deg} / \mathrm{sec}$ and wind angles within $0.05 \mathrm{deg}$.

Future planned experiments include development and demonstration of flight control technologies such as autorecovery algorithms, reconfigurable control laws, onboard fault detection, and adaptive control systems. Ultimately, testing of control mitigation systems for failure and damage configurations will be conducted.

\section{Summary}

Researchers at NASA LaRC are developing an experimental flight test capability based on a dynamically scaled transport aircraft. This capability will enable flight test validation of experimental technologies in support of the goals of NASA's Aviation Safety Program. The major components of the testbed are a dynamically scaled subscale flight vehicle, a ground based control and data system, and the facility at which the experiments will be conducted. This testbed fills a void in experimental capability between existing wind tunnels and full scale flight research. Used together, these three test techniques, along with simulation capabilities, will provide the resources to advance aircraft safety and robustness for the air-traveling public.

\section{References}

\footnotetext{
${ }^{1}$ Croft, J. W., "Refuse to Crash - NASA Tackles Loss of Control,” Aerospace America, March 2003.

${ }^{2}$ Belcastro, Celeste M.; Belcastro, Christine M.; “On the Validation of Safety Critical Aircraft Systems, Part II: An Overview of Experimental Methods”, AIAA Guidance, Navigation, and Control Conference, AIAA, Washington, DC, 2003.

${ }^{3}$ Jordan, T.L., Langford, W.M., and Hill, J.S., “Airborne Subscale Transport Aircraft Research Testbed, Aircraft Model Development”, AIAA 2005-6432, AIAA Guidance, Navigation, and Control Conference, AIAA, Washington, DC, 2005.
} 
${ }^{4}$ Bailey, R.M., Hostetler, R.W., Barnes, K.N., Belcastro, Christine M., and Belcastro, Celeste M., "Experimental Validation: Subscale Aircraft Ground Facilities and Integrated Test Capability,” AIAA Guidance, Navigation, and Control Conference, AIAA, San Francisco, CA, 2005.

${ }^{5}$ Gainer, T.G. and Hoffman, S. "Summary of Transformation Equations and Equations of Motion Used in Free-Flight and Wind Tunnel Data Reduction and Analysis,” NASA SP-3070, 1972.

${ }^{6}$ Wolowicz, C.H., Bowman, Jr., J.S., and Gilbert, W.P., "Similitude Requirements and Scaling Relationships as Applied to Model Testing”, NASA TP-1435, 1979.

${ }^{7}$ Foster, John V. et al; "Dynamics Modeling and Simulation of Large Transport Airplanes in Upset Conditions, AIAA-20055933, AIAA Guidance, Navigation and Control Conference, AIAA, San Francisco, CA, 2005.

${ }^{8}$ Belcastro, C.M.; "Overview of the Systems and Airframe Failure Emulation Testing \& Integration (SAFETI) Laboratory at the NASA Langley Research Center”, Proceedings of the International Conference on Lightning and Static Electricity, 2001.

${ }^{9}$ Van Vuren, R.J.; “Model Aircraft Operating Standards”, Advisory Circular AC 91-57, Dept. of Transportation, Fed. Aviation Administration, Washington, DC, June 1981.

${ }^{10}$ McGraw, J.W.; "Unmanned Aircraft Systems Operations in the U.S. National Airspace System - Interim Operational Approval Guidance”, AFS-400 UAS Policy 05-01, Dept. of Transportation, Fed. Aviation Admin., Washington, DC, September 2005.

${ }^{11}$ Shah, Gautam H., et al, "Wind-Tunnel Investigation of Commercial Transport Aircraft Aerodynamics at Extreme Flight Conditions”, SAE 2002-01-2912, World Aviation Congress \& Display, November 5-7, 2002.

${ }^{12}$ Aerodynamic Modeling of Large Transports for Upset Conditions: 2004 EURS Progress Report for NAS1-00106, November 3, 2004.

${ }^{13}$ Cunningham, Kevin, Foster, John V., Shah, Gautam H., Stewart, Eric C., Rivers, Robert A., Wilborn, James E., and Gato, William, "Simulation Study of a Commercial Transport Airplane During Stall and Post-Stall Flight”, 2004 SAE World Aviation Congress, SAE 2004-01-3100, Nov. 2-4, 2004.

${ }^{14}$ Cunningham, Kevin, et al, "Simulation Study of Flap Effects on a Commercial Transport Airplane in Upset Conditions", AIAA-2005-5908, AIAA Atmospheric Flight Mechanics Conference, AIAA, San Francisco, CA, 2005.

${ }^{15}$ Morelli, E.A.; "Global Nonlinear Aerodynamic Modeling Using Multivariate Orthogonal Functions”, AIAA Journal of Aircraft, Vol. 32, No. 2, March-April 1995. 